\title{
Phenotypic overlap between pyruvate dehydrogenase complex deficiency and FOXG1 syndrome
}

\author{
Yuichi Akaba ${ }^{1}$, Satoru Takahashi ${ }^{1}$, Ryo Takeguchi ${ }^{1}$, Ryosuke Tanaka ${ }^{1}$, Shin Nabatame ${ }^{2}$, \\ Hirotomo Saitsu ${ }^{3}$, and Naomichi Matsumoto ${ }^{4}$ \\ ${ }^{1}$ Asahikawa Medical University \\ ${ }^{2}$ Osaka University School of Medicine Graduate School of Medicine \\ ${ }^{3}$ Hamamatsu University School of Medicine \\ ${ }^{4}$ Yokohama City University Graduate School of Medicine
}

September 14, 2020

\begin{abstract}
Pyruvate dehydrogenase complex (PDHC) deficiency is a mitochondrial disorder. We report two cases of PDHC deficiency with clinical symptoms and brain imaging findings reminiscent of FOXG1 syndrome, suggesting a phenotypic overlap of these disorders.
\end{abstract}

Phenotypic overlap between pyruvate dehydrogenase complex deficiency and FOXG1 syndrome

Yuichi Akaba ${ }^{1}$, Satoru Takahashi ${ }^{1}$, Ryo Takeguchi ${ }^{1}$, Ryosuke Tanaka ${ }^{1}$, Shin Nabatame $^{2}$, Hirotomo Saitsu ${ }^{3}$, Naomichi Matsumoto ${ }^{4}$

1 Department of Pediatrics, Asahikawa Medical University, Asahikawa, Japan

2 Department of Pediatrics, Graduate School of Medicine, Osaka University, Osaka, Japan.

3 Department of Biochemistry, Hamamatsu University School of Medicine, Hamamatsu, Japan.

4 Department of Human Genetics, Graduate School of Medicine, Yokohama City University, Japan

*Corresponding author

Yuichi Akaba, MD

Department of Pediatrics, Asahikawa Medical University

2-1-1-1 Midorigaoka-Higashi,

Asahikawa, Hokkaido 078-8510, Japan

Tel: +81-166-68-2483, Fax: +81-166-68-2489

E-mail:akaba5p@asahikawa-med.ac.jp

Keywords

PDHC deficiency, FOXG1 syndrome, phenotype, MRI

Key Clinical Message 
Pyruvate dehydrogenase complex (PDHC) deficiency is a mitochondrial disorder. We report two cases of PDHC deficiency with clinical symptoms and brain imaging findings reminiscent of FOXG1 syndrome, suggesting a phenotypic overlap of these disorders.

\section{Introduction}

Developmental encephalopathy (DE) is associated with developmental impairment, intellectual disability, and other neurological disorders such as epilepsy and movement disorders. ${ }^{1}$ DE has developmental consequences arising directly from the effect of genetic variants. The clinical features of patients with DE overlap with those of many neurodevelopmental disorders, and one disorder is difficult to distinguish from the others.

FOXG1 syndrome is one of the DEs, caused by a loss-of-function variant of FOXG1, that encodes a brainspecific transcriptional factor critical for forebrain development. ${ }^{2,3}$ Patients with FOXG1 syndrome show microcephaly, hyperkinetic movement disorder, severe psychomotor delay without regression, and characteristic brain imaging findings. ${ }^{2,5}$ Because hyperkinetic movement disorders commonly develop by 1 year of age, they are proposed as a diagnostic marker for FOXG1 syndrome. ${ }^{5}$

Here, we report two cases of pyruvate dehydrogenase complex (PDHC) deficiency with clinical symptoms and brain imaging findings reminiscent of FOXG1 syndrome. They exhibited hyperkinetic involuntary movements and hand stereotypies, which are unusual for PDHC deficiency. ${ }^{6}$ This study demonstrates phenotypic overlap between PDHC deficiency and FOXG1 syndrome.

\section{Case presentation}

\subsection{Patient 1}

This 31-year-old female patient was born to non-consanguineous, healthy Japanese parents at 40 weeks of gestation after an uneventful pregnancy. Her birth weight and length were $2650 \mathrm{~g}(-1.1 \mathrm{SD})$ and $44.2 \mathrm{~cm}$ (2.8 SD), respectively. Her occipitofrontal circumference (OFC) was $32 \mathrm{~cm}$ (- $1.1 \mathrm{SD})$. She had no significant family medical history. Because of floppiness and inability to suck, she was fed through a nasogastric tube from the first day of life. At the age of 3 months, psychomotor delay, hypotonia, and strabismus were noted, and myoclonic seizures from the age of 11 months. The seizures were eventually controlled with phenytoin and phenobarbital. Developmental milestones were severely delayed; she acquired head control and turned over at 3 years, and was never able to walk unaided and never acquired speech sounds. She displayed hand stereotypies with hand-to-mouth movements. Her OFC was $43.5 \mathrm{~cm}(-6.6 \mathrm{SD})$ at 8 years, indicating that microcephaly had become more evident with time. Magnetic resonance imaging (MRI) scans of the brain showed ventricular dilatation and hypoplasia of the frontal lobe and the corpus callosum (Figure 1a and b). Based on the clinical and brain MRI features, FOXG1 syndrome was initially suspected. However, genetic testing identified a disease-associated variant in PDHA1 but not in FOXG1. Metabolic investigations revealed increased levels of lactate $(25.1 \mathrm{mg} / \mathrm{dL})$ and pyruvate $(1.93 \mathrm{mg} / \mathrm{dL})$ in blood, leading to a definite diagnosis of PDHC deficiency. Lactate and pyruvate accumulation were reduced to 12.9 and $1.06 \mathrm{mg} / \mathrm{dL}$ respectively, by administration of thiamine at a dose of $150 \mathrm{mg}$ a day; however, her neurological condition did not improve.

\subsection{Patient 2}

This female patient, aged 8 years, was born to non-consanguineous, healthy Japanese parents at 41 weeks of gestation after an uneventful pregnancy. Her birth weight and OFC were $3156 \mathrm{~g}(0.1 \mathrm{SD})$ and $32.0 \mathrm{~cm}$ (1.2 SD), respectively. She had no significant family medical history. During the neonatal period, she showed hypotonia, sleep disturbance, and inconsolable crying. The developmental milestones were severely delayed with no regression; she acquired head control at 6 months, turned over at 15 months, and sat up at 2 years. Because of feeding problems due to swallowing difficulties, she was fed via a nasogastric tube from 1 year and 9 months. Her head growth was postnatally decelerated and microcephaly became evident (OFCs of 40.2, 41.0 , and $43.0 \mathrm{~cm}$ at 7 months, 1 year, and 2 years, respectively, all below $-2 \mathrm{SD}$ ). At the age of 3 years, she remained incapable of standing up, acquiring speech sounds, and purposeful hand skills. She displayed prominent hyperkinetic movement disorders with hand stereotypies. Brain MRI scans showed microcephaly 
with hypoplasia of the frontal lobe and the corpus callosum (Figure 1c and d). She showed clinical features and brain MRI findings that were reminiscent of FOXG1 syndrome; however, she had increased lactate and pyruvate levels in blood (lactate $18.2 \mathrm{mg} / \mathrm{dL}$, pyruvate $2.5 \mathrm{mg} / \mathrm{dL}$ ) and cerebrospinal fluid (CSF) (lactate $32.6 \mathrm{mg} / \mathrm{dL}$, pyruvate $3.3 \mathrm{mg} / \mathrm{dL}$ ), although the lactate/pyruvate molar ratio was normal. Genetic testing revealed a disease-associated variant of PDHA1, leading to a diagnosis of PDHC deficiency. She was enrolled in a clinical trial of pyruvate therapy for mitochondrial diseases, ${ }^{7}$ which was effective in promoting motor development, but not the cognitive impairment. She was able to walk a few steps at 7 years of age, but remained unable to speak any meaningful words.

\subsection{Identification of $P D H A 1$ variants}

Blood samples were collected from the patients and their parents after obtaining written informed consent from the parents. This study was approved by the Committee for Ethical Issues at Asahikawa Medical University. We performed whole exome sequencing in patient 1 as previously described ${ }^{8}$ and direct sequencing of PDHA1 in patient 2 and, in both, identified a same frameshift variant in PDHA1, NM_000284.3:c.934_940del, p.(Ser312Valfs*12) (Figure 2). Testing of the patients' parents confirmed that thePDHA1 variant emerged de novo .

\section{Discussion}

PDHC catalyzes the oxidative decarboxylation of pyruvate to acetyl-CoA, a critical step in energy production. The clinical spectrum of PDHC deficiency ranges from episodic ataxia to severe fatal neonatal lactic acidosis. ${ }^{9}$ We identified a disease-associated variant in the $\mathrm{X}$-linked PDHA1 in two female patients who were clinically suspected to have FOXG1 syndrome. Both had the same heterozygous deletion of a 7-bp repeat sequence in exon 10 of PDHA1. This mutation was already reported in female patients with PDHC deficiency who exhibited variations in clinical severity, most probably due to different patterns of X-chromosome inactivation. ${ }^{10}$ Our patients exhibited similar clinical features, including microcephaly, hypotonia, feeding difficulties, abnormal involuntary movements, stereotypic hand movements, and a profound delay in psychomotor development. Brain MRI scans revealed cerebral atrophy, ventriculomegaly, and hypoplasia of the corpus callosum. These features have been reported in patients with FOXG1 syndrome, ${ }^{11-13}$ suggesting a phenotypic overlap between PDHC deficiency and FOXG1 syndrome (Table 1).

Although patients with PDHC deficiency shared clinical features with FOXG1 syndrome, specific brain MRI findings may distinguish PDHC deficiency from FOXG1 syndrome. Corpus callosum hypogenesis was observed in both diseases. However, the corpus callosum in FOXG1 syndrome was affected mostly the anterior parts ${ }^{14}$ whereas in PDHC deficiency, the posterior body and splenium were preferentially affected. ${ }^{15}$ Moreover, patients with PDHC deficiency had dilated ventricles with ventricular septations and/or subependymal cysts (Figure 1), probably due to a destructive process secondary to a metabolic defect, which were absent in FOXG1 syndrome. Subependymal cysts were also described in other metabolic disorders that cause primary lactic acidosis. ${ }^{16}$ Intraventricular septations probably reflect the evolution of subependymal cysts. Thus, none of the MRI findings described here is unique to PDHC deficiency. The detection of these MRI findings should prompt further investigation, including metabolic analysis and genetic testing for PDHA1 variants.

Most importantly, PDHC deficiency is commonly associated with lactic acidosis. ${ }^{9}$ Specific structural brain abnormalities, with the characteristic clinical features may allow distinction of PDHC deficiency from FOXG1 syndrome if the lactate and pyruvate levels are elevated in the blood and/or CSF. Therefore, this study further reinforces the necessity of measuring the lactate and pyruvate levels in blood and/or CSF even in the cases of clinically suspected FOXG1 syndrome.

\section{Acknowledg \soutements}

We thank all the patients and their family members. The permission was obtained from the patient's parents for presentation.

\section{Ethical approval}


Institutional approval was obtained. No identifiable patient information was presented in this report.

\section{Conflict of Interest Disclosures}

None declared.

\section{Funding information}

This work was supported in part by Japan Agency for Medical Research and Development (AMED) grants under the numbers JP20ek0109486, JP20ek0109301, JP20ek0109348 and JP20kk0205012 (to NM.), and Japan Society for the Promotion of Science (JSPS) KAKENHI JP17H01539 (to NM.).

\section{Author contributions}

YA: contributed to collecting all the clinical data and writing of the draft manuscript. ST: contributed to literature review and revision of the manuscript. RT, RT, SN, HS, and NM critically reviewed the manuscript. All Authors approved the contents of the submitted manuscript.

\section{References}

[1] Scheffer IE, Berkovic S, Capovilla G, et al. ILAE classification of the epilepsies: Position paper of the ILAE Commission for Classification and Terminology. Epilepsia . 2017;58:512-521.

[2] Ariani F, Hayek G, Rondinella D, et al. FOXG1 is responsible for the congenital variant of Rett syndrome. Am J Hum Genet . 2008;83:89-93.

[3] Wong LC, Singh S, Wang HP, Hsu CJ, Hu SC, Lee WT. FOXG1-Related Syndrome: From Clinical to Molecular Genetics and Pathogenic Mechanisms.Int J Mol Sci . 2019: 20:e4176.

[4] Bahi-Buisson N, Nectoux J, Girard B, et al. Revisiting the phenotype associated with FOXG1 mutations: two novel cases of congenital Rett variant. Neurogenetics . 2010;11:241-249.

[5] Caporali C, Signorini S, Giorgis VD, Pichiecchio A, Zuffardi O, Orcesi S. Early-onset movement disorder as diagnostic marker in genetic syndromes: Three cases of FOXG1-related syndrome. Eur Paediatr Neurol . 2018;22:336-339.

[6] Patel KP, O'Brien TW, Subramony SH, Shuster J, Stacpoole PW. The spectrum of pyruvate dehydrogenase complex deficiency: clinical, biochemical and genetic features in 371 patients. Mol Genet Metab . 2012;105:34-43.

[7] Koga Y, Povalko N, Katayama K, et al. Beneficial effect of pyruvate therapy on Leigh syndrome due to a novel mutation in PDH E1 $\alpha$ gene. Brain Dev . 2012;34:87-91.

[8] Sakamoto M, Kouhei D, Haniffa M, et al. A novel ITPA variant causes epileptic encephalopathy with multiple-organ dysfunction. J Hum Genet . 2020;65:751-757.

[9] Brown GK. Pyruvate dehydrogenase E1 $\alpha$ deficiency. J Inherit Metab Dis . 1992;15:625-633.

[10] Lissens W, De meirleir L, Seneca S, et al. Mutations in the X-linked pyruvate dehydrogenase (E1) alpha subunit gene (PDHA1) in patients with a pyruvate dehydrogenase complex deficiency. Hum Mutat . 2000;15:209-219.

[11] Kortüm F, Das S, Flindt M, et al. The core FOXG1 syndrome phenotype consists of postnatal microcephaly, severe mental retardation, absent language, dyskinesia, and corpus callosum hypogenesis. $J$ Med Genet . 2011;48:396-406.

[12] Takahashi S, Matsumoto N, Okayama A, et al. FOXG1 mutations in Japanese patients with the congenital variant of Rett syndrome. Clin Genet . 2012;82:569-573.

[13] Kumakura A, Takahashi S, Okajima K, Hata D. A haploinsufficiency of FOXG1 identified in a boy with congenital variant of Rett syndrome. Brain Dev . 2014;36:725-729. 
[14] Vegas N, Cavallin M, Maillard C, et al. Delineating FOXG1 syndrome: From congenital microcephaly to hyperkinetic encephalopathy.Neurol Genet . 2018;4:e281.

[15] Ah Mew N, Loewenstein JB, Kadom N, et al. MRI features of 4 female patients with pyruvate dehydrogenase E1 alpha deficiency.Pediatr Neurol . 2011;45: 57-59.

[16] Dey R, Mine M, Desguerre I, et al. A new case of pyruvate dehydrogenase deficiency due to a novel mutation in the PDX1 Gene.Ann Neurol . 2003:53:273-277.

Table 1 Phenotypic overlap between PHDC deficiency and FOXG1 syndrome

\begin{tabular}{|c|c|c|c|}
\hline & Patient 1 & Patient 2 & $\begin{array}{l}\text { FOXG1 syndrome } \\
(\mathrm{n}=29)^{\mathrm{a}}\end{array}$ \\
\hline Sex & female & female & 21 females; 8 males \\
\hline \multicolumn{3}{|l|}{ Growth } & 10 months to 34 years \\
\hline Failure to thrive & $(+)$ & $(+)$ & $21 / 22$ \\
\hline \multicolumn{3}{|l|}{ Development } & $24 / 24$ \\
\hline Intellectual disability & $(+)$ & $(+)$ & $29 / 29$ \\
\hline Absent language & $(+)$ & $(+)$ & $27 / 28$ \\
\hline Sitting & $(-)$ & $(+)$ & $6 / 26$ \\
\hline Walking & $(-)$ & $(-)$ & $2 / 29$ \\
\hline \multicolumn{4}{|l|}{ Motor and dyskinesias } \\
\hline Hypotonia & $(+)$ & $(+)$ & $19 / 19$ \\
\hline Involuntary movements & $(+)$ & $(+)$ & $21 / 23$ \\
\hline Stereotypic movements & $(+)$ & $(+)$ & $11 / 14$ \\
\hline Strabismus & $(+)$ & $(-)$ & $11 / 12$ \\
\hline Bruxism & $(-)$ & $(+)$ & $18 / 24$ \\
\hline Epilepsy & $(+)$ & $(-)$ & $20 / 28$ \\
\hline Feeding difficulties & $(+)$ & $(+)$ & $15 / 20$ \\
\hline Brain MRI & & & \\
\hline $\begin{array}{l}\text { Hypoplasia of corpus } \\
\text { callosum }\end{array}$ & $(+)$ & $(+)$ & $21 / 23$ \\
\hline
\end{tabular}

${ }^{\text {a }}$ Clinical features of 29 patients with FOXG1 syndrome have previously reported. ${ }^{11-13}$

\section{Figure legends}

\section{Figure 1}

(a-d) Images obtained when patient 1 (a and b) and patient 2 (c and d) were 29 and 3 years old, respectively. T1- and T2-weighted images of patients with PDHC deficiency show asymmetrical ventriculomegaly with subependymal cysts (a, arrows), ventricular septation (c, arrow), and hypoplasia of the corpus callosum (b and d).

\section{Figure 2}

Automated DNA sequencing with the polymerase chain reaction product from Patients 1 and 2 showed a heterozygous deletion of a 7-bp repeat sequence (AGTAAGA) in exon 10 of PDHA1(NM_000284.3:c.934_940del), which resulted in a reading frame shift and introduction of a premature stop codon (p.Ser312Valfs*12).

\section{Hosted file}


Fig.1 (CCR).pptx available at https://authorea.com/users/358574/articles/480689-phenotypicoverlap-between-pyruvate-dehydrogenase-complex-deficiency-and-foxg1-syndrome

\section{Hosted file}

Fig.2 (CCR).pptx available at https://authorea.com/users/358574/articles/480689-phenotypicoverlap-between-pyruvate-dehydrogenase-complex-deficiency-and-foxg1-syndrome 